\title{
An AI Approach to Locate Cluster Centre in Wireless Sensor Networks
}

\author{
Sandhya R \\ Research Scholar \\ Bharathiar University \\ Coimbatore \\ sandhyakishore32@gmail.com
}

\author{
Dr. N Sengottaiyan \\ Director, Academics \\ Hindustan College of Engg.and Technology, \\ Coimbatore, Tamil Nadu 641032 \\ nsriram3999@gmail.com
}

\begin{abstract}
Wireless sensor networks, which consist of a immense number of sensor nodes have emerged as an inevitable, effective and important technology in telecommunication field. Sensor nodes can be deployed in any harsh environment for health monitoring, industrial monitoring, structural analysis, detecting intruders etc. Since it works in battery, the major challenge is to improve the lifetime of sensor node by effectively reducing energy consumption. Otherwise, it is very difficult to frequently replace the sensor nodes. For this, many routing protocols have been proposed in which cluster based routing protocol plays a vital role. In cluster based routing protocol, entire WSN network is divided into several groups or clusters. Then for each cluster a cluster head $(\mathrm{CH})$ is selected. Sensors sense the data and forward it to their corresponding $\mathrm{CH}$ where the entire data of that cluster get aggregated and transmitted to base station (BS). The clustering technique offers more benefit than existing flat based routing protocols since the data is transmitted via $\mathrm{CH}$ rather than directly by each sensor nodes. Thus the selection of $\mathrm{CH}$ is very important. Here we proposed a method named firefly based nearest neighbor chain algorithm to select the centre of cluster based on which $\mathrm{CH}$ selection can be done.
\end{abstract}

Index Terms-WSN; energy consumption; cluster based routing protocol; $\mathrm{CH}$ selection.

\section{INTRODUCTION}

Developments in electronics field paved the way for introducing low cost, small sized and easily deployable sensors which became an important computing technology for environment monitoring. WSN which consists of a huge number of sensor nodes is not only a simple sensing network but also storage, processing and efficient communication network. It provides services in healthcare, structure and environment monitoring [1]. Sensor nodes collect data and send to $\mathrm{BS}$ via $\mathrm{CH}$. It is tedious task to frequently replace the battery once a sensor node is deployed. So it is necessary to find out a technique that will reduce the energy consumption of sensor nodes for improving the network life time.

Based on network structure, the routing protocols in WSN are classified in to flat based routing protocol and cluster based routing protocol [2]. In flat based routing protocol, each sensor node independently forwards the data to BS irrespective of its position. But in cluster based routing method, data sensed by each sensor node is collected by corresponding $\mathrm{CH}$ and then forwarded to BS. This will reduce the overall communication overhead and energy consumption.

Some protocols adopt single hop communication whereas others adopt multihop communication to transfer the data to BS [3]. CH selection is an important step in cluster based routing protocol. Normally $\mathrm{CH}$ is selected based on energy, centrality etc. It is the $\mathrm{CH}$ which aggregates the data in the network and forwarded the same to the destination. It is better to select a node located at the centre of cluster as $\mathrm{CH}$. This is because; other nodes can communicate with this cluster head with minimal energy.

Here in this paper, we are integrating nearest neighbor chain algorithm with firefly algorithm to find the centre of clusters with the most optimal neighbour nodes present. For this we have to calculate the distance between each sensor nodes and attractiveness of firefly. Using an equation we can calculate movement of firefly which is attracted to another firefly. Algorithm works for several iterations. Distance and movement of the firefly can be updated at each iteration.

This paper is organized as follows: Section II describes related works that inspired and motivates us to this work. Section III explains proposed firefly based nearest neighbour algorithm to find the centre of cluster and section IV concludes the paper with future enhancements.

\section{RELATED WORK}

Different cluster based routing protocols with energy efficiency have been proposed by researches to improve and enhance the network longevity of wireless sensor network.

The first clustering protocol named Low Energy Adaptive Clustering Hierarchy (LEACH) [4] protocol uses a distributed clustering algorithm in which $\mathrm{CHs}$ are selected based on a predetermined probability. Each other nodes select their cluster based on the distance to $\mathrm{CH}$. But leach does not provide a uniform distribution of cluster head and sometimes same nodes became $\mathrm{CH}$ in more than one round repeatedly. This will abruptly reduce the energy of that particular node and creates an energy hole. Hybrid Energy-Efficient Distributed clustering (HEED protocol) 
[5], which uses energy and communication cost for cluster head formation restricts the selection of two nodes within the same transmission coverage. It provides flexible $\mathrm{CH}$ selection and inter-CH connectivity. This protocol introduces an extra communication overhead or cost since each node should constantly communicate with its neighbours for specific number of rounds. Thus it is uncomfortable large -scale networks.

Another protocol named Distributed Energy Efficient Clustering (DEEC) [6] proposed by researchers selects $\mathrm{CH}$ based on the probability of ratio of residual energy to the average energy of the network. It computes the reference energy that each node expend based on which a value of network lifetime is estimated. It induces an overhead in the network topology and failed to calculate the average energy precisely. LEA CH-C [7], enhancement fro $\mathrm{m}$ LEA CH adopts a centralized approach by sending its id, position and energy value to the $\mathrm{BS}$ for $\mathrm{CH}$ selection.

PEGASIS [8] proposed by S.Lindsey suffers delay for forming a chain to transfer the data to BS. It requires a complete overview of network. This method constructs chain instead of clusters and selects a leader for that chain. Nodes will transfer the data through the chain to the leader and later leader will forward the aggregated data to BS. TEEN [9] proposed by A.Manjeshwar, et al; will reports data only when the sensed attribute reaches a particular threshold. Thus it can't be used for periodic report application. As a solution to this, they later implemented APTEEN [10] which can be used in proactive and reactive conditions.

The paper proposed by Jung et al. mention CCS [11] in which an integrated approach of PEGASIS and clusterbased method is formulated. Here the entire network is divided into various clusters and in each cluster a chain is formed by selecting a leader without considering the residual energy. It makes unbalanced distribution of nodes in each cluster. TL-LEACH introduced by Loscrì et al. [12] suggests a two-level hierarchy where there is primary $\mathrm{CH}$ and secondary $\mathrm{CH}$ compete themselves. Here selection of $\mathrm{CH}$ is done without considering residual energy of nodes. A lot of message passing introduces communication overhead and affects network lifetime.

EECS proposed by Ye et al. [13] needs global knowledge about distance between $\mathrm{BS}$ and each $\mathrm{CH}$ for making the network more energy efficient. Here the nodes compete themselves to beat others and win as $\mathrm{CH}$. For this, each node send its residual energy to their neighbours and choose the one with highest energy as CH. LEACH-M [14] proposed by D. S. Kim, et.al; introduces node mobility which results in large number of packet losses while moving $\mathrm{CH}$ before selecting a new $\mathrm{CH}$ for the next iteration.

Base-Station Controlled Dynamic Clustering Protocol (BCDCP), proposed by Muruganathan et al. [15], is not suitable for large distance communication since it uses single hop routing strategy with centralized approach. In [16], Wu et al; introduces a sleep/wake scheduling protocol that preserves energy by keeping the radio transceivers to sleep periodically. But it suffers scheduling and synchronizing overhead.

To improve the efficiency of clustering and to mitigate the energy consumption problem we propose a new method to determine the centre of cluster for selecting a best $\mathrm{CH}$. This approach uses combination of firefly algorithm and nearest neighbour method to effectively choosing the centroid of cluster. The attractiveness of fly is calculated and updated in each round. Also we are calculating the distance between each node by using Euclidean distance formula. Based on both theses parameters a best values is selected as cluster centre.

\section{FIREFLy BASED NEAREST NEIGHBOUR CHAIN ALGORITHM}

Sensor nodes which are battery operated are most often constrained in energy due to difficulty and inability to replace or recharging the nodes. Thus one of the most challenges in designing a protocol is energy consumption. A WSN network should be self-organized and distributed by which each sensor node can detect changes in the environment effectively. With respect to flat based and location based routing protocol, the cluster based routing protocols provides much longer network longevity. Here the entire network is divided into several numbers of clusters and one node from each cluster is selected as CH.CH collects data from each cluster, aggregates it and forwarded to the sink. The major role in clustering is to divide the entire network into groups and select suitable $\mathrm{CH}$. Here we are suggesting a method to find the centre of cluster based on which a $\mathrm{CH}$ can be selected.

The proposed method uses combination of firefly algorithm with nearest neighbour approach to find the centroid of cluster. Each iteration attractiveness of fly and distance between nodes are calculated and updated. At last, we select most suitable value as centre of cluster based on which $\mathrm{CH}$ is selected.

Two main parameters are considered in this approach: attractiveness and distance. Attractiveness implies the similarity of nodes present in the network. Distance between nodes is calculated based on the Cartesian distance of the nodes using Euclidean formula. In this phase, we combined firefly algorithm with nearest neighbour chain algorithm to find the centre of cluster with most optimal neighbour nodes present in the topology.

The attractiveness of the firefly can be calculated as:

$$
\beta(r)=\beta_{0} e^{-\gamma r^{2}}
$$

where $\beta_{0}$ is the attractiveness at $r=0$ and $\gamma$ is the light absorption coefficient. 
The Cartesian distance between any two sensor nodes can be determined by:

$$
D(x, y)=\sqrt{\left(x_{i}-x_{j}\right)^{2}+\left(y_{i}-y_{j}\right)^{2}}
$$

where $x_{i}$ and $y_{i}$ denotes the $(x, y)$ coordinates of node $i$ whereas $x_{j}$ and $y_{j}$ denotes the $(x, y)$ coordinates of node $j$.

The movement of the firefly $i$, which is attracted to firefly $\mathrm{j}$ is estimated by:

$$
X_{i}=x_{i}+\beta_{0} e^{-r^{2}}\left(x_{j}-x_{i}\right)+\alpha(\text { rand }-0.5)
$$

Objective function which is used to find the centre of the clusters with the proposed FNNC algorithm can be formulated as follows

Objective function $=\operatorname{Dis}(X, Y)=\sqrt{\sum_{i=1}^{d}\left(X_{p i}-Y_{j i}\right)}$

where $X_{p i}$ is the $p^{\text {th }}$ data path vector and $Y_{j i}$ is the distance vector of cluster $\mathrm{j}$.

The aim is to find minimum value for objective function after the entire iteration completed. During each iterat ion, the movement and distance of the firefly gets updates.

Distance can be updated by using the formula:

$$
D\left(x_{\text {best }}, y_{\text {best }}\right)=\sqrt{\left(x_{i}-x_{\text {gbest }}\right)^{2}+\left(y_{i}-y_{\text {gbest }}\right)^{2}}
$$

Updation of firefly movement can be expressed as:

$$
X_{i+1}=x_{i}+\beta_{0} e^{-r^{2}}\left(x_{j}-x_{i}\right)+\beta_{0} e^{-x_{i, g b e t s}^{2}}\left(x_{\text {gbest }}-x_{i}\right)+\alpha(\text { rand }-0.5)
$$

The steps in the proposed clustering algorithm can be explained as follows:

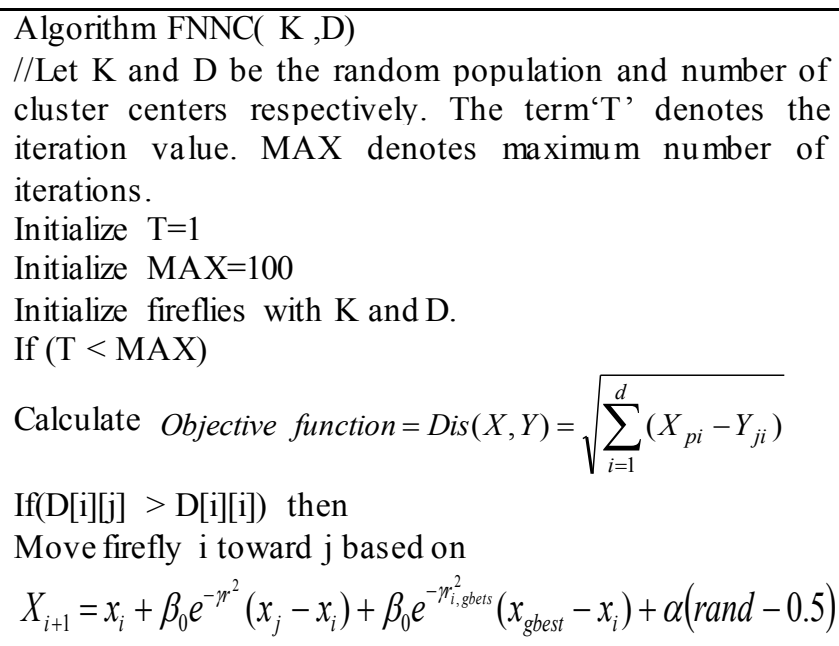
cluster centers respectively. The term' $T$ ' denotes the iteration value. MAX denotes maximum number of iterations.

Initialize $\mathrm{T}=1$

Initialize $\mathrm{MAX}=100$

Initialize fireflies with $\mathrm{K}$ and $\mathrm{D}$.

If $(\mathrm{T}<\mathrm{MAX})$

Calculate Objective function $=\operatorname{Dis}(X, Y)=\sqrt{\sum_{i=1}^{d}\left(X_{p i}-Y_{j i}\right)}$

If(D[i][i] $>D[i][i])$ then

Move firefly $\mathrm{i}$ toward $\mathrm{j}$ based on

$X_{i+1}=x_{i}+\beta_{0} e^{-r^{2}}\left(x_{j}-x_{i}\right)+\beta_{0} e^{-r_{i, g b e t s}^{2}}\left(x_{\text {gbest }}-x_{i}\right)+\alpha($ rand -0.5$)$

to update the locations

End if

$\mathrm{T}=\mathrm{T}+1$

End if

Algorithm 1: Steps in FNNC Algorithm

From the above proposed algorithm, the following inference is made:

The most important parameter in firefly algorithm is $\gamma$ (Absorption coefficient), it plays very crucial role in determining the speed of convergence and how FA algorith $m$ behaves. Theoretically, $\gamma \in[0, \infty)$ but in actual practice, the value of $\gamma$ is taken as 1 . So for almost all the applications it varies from 0.01 to 10 . Light intensity $I_{i}$ of a firefly $u_{i}$ at location $x_{i}$ is determined by the objective function. For our proposed method, firefly and the location express in terms of node and location of the node in WSN respectively.

\section{CONCLUSION AND FUTURE WORKS}

Cluster based routing protocol plays a major role in WSN communication. This type of network topology is easier to implement, manage and deploy. Data aggregation at each $\mathrm{CH}$ reduces redundancy and mitigates the communication overhead. The major concern is to select a node as $\mathrm{CH}$ for communication to the BS. This paper mainly focused on how to find out the centre of cluster for selecting a $\mathrm{CH}$. Because an efficient selection $\mathrm{CH}$ makes the communication overhead lower and improve the network lifetime. The proposed method uses firefly algorith $m$ with nearest neighbour chain method to calculate the centroid. It achieves the aim by considering two parameters: attractiveness and distance. At each iteration, attractiveness or firefly movement and distance of nodes get updated. We are comparing the global best value with current best and initialize cluster centre with global best value. This process continues until maximum iteration reached.

As future work, Genetic Algorithm based Harmony Search algorithm can be implemented for optimal $\mathrm{CH}$ selection. Here in itially the entire nodes can be considered as population for which we are calculating fitness value. Then those which having highest fitness values are taken and crossover each other. By using this method best nodes can be selected as $\mathrm{CH}$.

\section{REFERENCES}

[1] Zheng J., Jamalipour A., "Wireless sensor networks a networking perspective", IEEE book, John Wiley \& Sons, 2009

[2] Khamfroush H., Saadat R., Khademzadeh A., Khamfroush K., "Life time increase for wireless sensor networks using cluster based routing", International Association of Computer Science and InformationTechnology-Spring Conference(IACSIT SC),2009, pp. 14-18. 
[3] Wang G., Zhu H., Dai H., Wu L., Xiong B., "The cluster algorithm of wireless sensor networks based on multihop between clusters", Computer Science and Information Engineering, vol. 3, 2009, pp. $177-181$.

[4] W. Heinzelman, A. Chandrakasan, H. Balakrishnan, EnergyEfficient Communication protocol for wireless microsensor networks, in the Proceedings of the 33rd International Conference on System Science(HICSS00), Hawaii, U.S.A., January 2000.

[5] Younis, O.; Fahmy, S. "HEED: A hybrid, energy-efficient, distributed clustering approach for ad- hoc sensor networks". IEEE Trans. Mobile Comput. 2004, 3, 366-379.

[6] L. Qing, Q. Zhu, and M. Wang, "Design of a distributed energyefficient clustering algorithm for heterogeneous wireless sensor networks," Comput.Commun., vol. 29, no. 12, pp. 2230-2237, Aug. 2006.

[7] X. H. Wu, S. Wang, "Performance comparison of LEACH and LEACH-C protocols by NS2," In Proceedings of 9th International Symposium on Distributed Computing and Applications to Business, Engineering and Science. Hong Kong, China, pp. 254258,2010

[8] S. Lindsey, C.Raghavendra, "PEGASIS: Power-Efficient Gathering in Sensor Information Systems," In Proc. IEEE Aerospace Conference, USA, Montana, Vol. 3, pp. 1125-1130, 2002.

[9] A. Manjeshwar, D. Agrawal, "TEEN: A Routing Protocol for Enhanced Efficiency in Wireless Sensor Networks," In Proc. 15th InternationalParallel and Distributed Processing Symposium (IPDPS'01) Workshops, USA, California, 2001, pp. 2009-2015.
[10] Manjeshwar, A.; Agrawal, D. P. "APTEEN: A Hybrid Protocol for Efficient Routing and Comprehensive Information Retrieval in Wireless Sensor Networks." In Proceedings of the 2nd International Workshop on Parallel and Distributed Computing Issues in Wireless Networks and Mobile computing, Lauderdale, FL, USA, 15-19 April 2002; pp. 195-202.

[11] Jung, S.; Han, Y.; Chung, T. "The Concentric Clustering Scheme for Efficient EnergyConsumption in the PEGASIS"; In Proceedings of the 9th International Conference on AdvancedCommunication Technology, Gangwon-Do, Kore ; pp. 260-265, February 2007.

[12] Loscri, V.; Morabito, G.; Marano, S.; "A Two-Level Hierarchy for Low-Energy Adaptive Clustering Hierarchy"; In Proceedings of the 2nd IEEE Semiannual Vehicular TechnologyConference, Dallas, TX, USA; pp. 1809-1813; September 2005.

[13] Ye, M.; Li, C.; Chen, G.; Wu, J. "An energy efficient clustering scheme in wireless sensor networks". Ad Hoc Sens. Wirel. Netw. 2006, 3, 99-119.

[14] D. S. Kim and Y. J. Chung, "Self-organization routing protocol supporting mobile nodes for wireless sensor network," in Proc.First International Multi-Symposiums on Computer and Computational Sciences, Hangzhou, China, 2006.

[15] Murugunathan, S. D.; Ma, D. C. F.; Bhasin, R. I.; Fapajuwo, A. O. "A Centralized Energy-Efficient Routing Protocol for Wireless Sensor Networks". IEEE Radio Commun. 2005, 43, S8-S13.

[16] Y. Wu, S. Fahmy, N. Shroff, "Energy Efficient Sleep/Wake Scheduling for Multi-Hop Sensor Networks: non-Convexity and Approximation Algorithm," In Proc. 26th Annual IEEE Conference on Computer Communications, Anchorage, Alaska pp. 1568-1576, 2007. 